\title{
BOUNDARY LAYERS IN APPROXIMATE SOLUTIONS
}

\author{
K. T. JOSEPH
}

\begin{abstract}
In this paper we study the development of boundary layers in parabolic approximate solutions of the initial boundary value problem for linear strictly hyperbolic systems of equations in one space variable. We also analyse the boundary layer behaviour.
\end{abstract}

\section{INTRODUCTION}

Let $D=\{(x, t): 0 \leq x \leq 1, t>0\}$ and let $A(x, t)$ be a smooth $m \times m$ matrix which has $m$ real distinct eigenvalues, $k$ of them negative and $(m-k)$ of them positive:

$$
\lambda_{1}(x, t)<\lambda_{2}(x, t)<\cdots<\lambda_{k}(x, t)<0<\lambda_{k+1}(x, t)<\cdots<\lambda_{m}(x, t) .
$$

Let $r_{j}(x, t)$ be a right eigenvector and $l_{j}(x, t)$ a left eigenvector corresponding to $\lambda_{j}(x, t)$.

It is well known (see Courant-Hilbert [3]) that a well-posed problem in $D$ for the strictly hyperbolic system

$$
u_{t}+A(x, t) u_{x}=0 \text {, }
$$

where $u \in R^{m}$, is to prescribe $u(x, 0)=u_{0}(x), l_{j}(0, t) u(0, t), j=k+1$, $\ldots, m$, and $l_{j}(1, t) u(1, t), j=1,2, \ldots, k$.

The corresponding parabolic problem is

$$
\begin{aligned}
& u_{t}^{\varepsilon}+A(x, t) u_{x}^{\varepsilon}=\varepsilon u_{x x}^{\varepsilon}, \\
& u^{\varepsilon}(x, 0)=u_{0}(x), \\
& u^{\varepsilon}(0, t)=u_{1}(t), \\
& u^{\varepsilon}(1, t)=u_{2}(t),
\end{aligned}
$$

where the data are compatible at the corners. Existence, uniqueness and smoothness of $u^{\varepsilon}$ are known (see A. Friedman [4]).

In $\S 2$ we study the limit as $\varepsilon$ tends to zero in (1.2). Since more boundary conditions are prescribed for parabolic equations than for hyperbolic equations, some boundary conditions get lost in the passage $\varepsilon \rightarrow 0$. This loss manifests

Received by the editors June 21, 1987 and, in revised form, January 12, 1988.

1980 Mathematics Subject Classification (1985 Revision). Primary 35B25; Secondary 35L50.

After this work was completed, the author learned from Professor Daniel Michelson's research announcement that he also considered a similar problem for several space variables. 
itself in the region of rapid change near $x=0$ and near $x=1$, called the boundary layer. Such problems, usually called in the literature singular perturbation problems, have been described by Lyusternik and Viski [6] for linear ode's and linear scalar elliptic equations, and by Bardos and Rauch [2] and Bardos, Brezis, Brezis [1] for maximal positive symmetric operators. In [2] Bardos and Rauch remark that one can prove $H^{s}$ convergence, $s<\frac{1}{2}$ for parabolic systems, even in several space variables using the same ideas but with some extra arguments.

Here we consider the case of one space variable and get results in the maximum norm. Our approach is different from Bardos and Rauch; we construct boundary layer functions near the boundaries and derive careful energy estimates to get uniform convergence. The construction of the boundary layer function is classical, at least in the scalar elliptic case and ode's (see Lyusternik and Visik [6]).

Before taking up the general case, we give two typical and simple examples illustrating possible loss of boundary conditions.

Example 1. Consider the following problem in $x>0, t>0$.

$$
u_{t}^{\varepsilon}+u_{x}^{\varepsilon}=\varepsilon u_{x x}, \quad u^{\varepsilon}(x, 0)=0, \quad u^{\varepsilon}(0, t)=u_{b}(t),
$$

$u_{b}(t)$ is smooth and compatible: $u_{b}(0)=0$. When $\varepsilon=0$, the problem is

$$
u_{t}^{0}+u_{x}^{0}=0, \quad u^{0}(x, 0)=0, \quad u^{0}(0, t)=u_{b}(t) .
$$

Set $e^{\varepsilon}(x, t)=u^{\varepsilon}(x, t)-u^{0}(x, t)$, then $e^{\varepsilon}(x, t)$ solves the following:

$$
\begin{gathered}
e_{t}^{\varepsilon}+e_{x}^{\varepsilon}=\varepsilon e_{x x}+\varepsilon u_{x x}^{0}, \\
e^{\varepsilon}(x, 0)=0, \quad e^{\varepsilon}(0, t)=0 .
\end{gathered}
$$

Let

$$
k=\sup _{x \geq 0}\left|u_{x x}^{0}(x, t)\right| .
$$

From the maximum principle for parabolic equations we conclude that

$$
\left|e^{\varepsilon}(x, t)\right| \leq \varepsilon k T \text { for } 0 \leq t \leq T,
$$

with shows that

$$
e^{\varepsilon}(x, t)=u^{\varepsilon}(x, t)-u^{0}(x, t) \rightarrow 0,
$$

uniformly in $x \geq 0,0 \leq t \leq T$.

Example 2. Now we consider the following problem in $x>0, t>0$.

$$
u_{t}^{\varepsilon}-u_{x}^{\varepsilon}=\varepsilon u_{x x}, \quad u^{\varepsilon}(x, 0)=0, \quad u^{\varepsilon}(0, t)=u_{b}(t),
$$

$u_{b}(t)$ is smooth and compatible: $u_{b}(0)=0$. When $\varepsilon=0$, the problem is

$$
u_{t}^{0}-u_{x}^{0}=0, \quad u^{0}(x, 0)=0,
$$

and no boundary conditions are needed. In fact $u^{0}(x, t)=0$, as is seen by the method of characteristics. 
For (*) we can get a closed form solution; set

$$
u^{\varepsilon}=e^{-x / 2 \varepsilon-t / 4 \varepsilon} v^{\varepsilon}(x, t),
$$

then from $(*)$ we obtain $v_{t}=\varepsilon v_{x x}, v(x, 0)=0, v(0, t)=u_{b}(t) e^{t / 4 \varepsilon}$, whose solution is

$$
v(x, t)=-\frac{2}{\sqrt{\pi}} \int_{0}^{t} \partial_{s}\left[\int_{x / 2 \sqrt{\varepsilon}(t-s)}^{\infty} e^{-y^{2}} d y\right]\left(e^{s / 4 \varepsilon} u_{b}(s)\right) d s .
$$

Hence

$$
\begin{aligned}
& u^{\varepsilon}(x, t)=\frac{2}{\sqrt{\pi}} e^{-x / 2 \varepsilon-t / 4 \varepsilon}\left[u_{b}(t) \int_{x / 2 \sqrt{\varepsilon} t}^{\infty} e^{-y^{2}} d y\right. \\
& \left.+\int_{0}^{t} \int_{x / 2 \sqrt{\varepsilon}(t-s)}^{\infty} e^{-y^{2}} d y \partial_{s}\left(e^{s / 4 \varepsilon} u_{b}(s)\right) d s\right],
\end{aligned}
$$

from which it is clear that

$$
\lim _{\varepsilon \rightarrow 0} \sup _{\substack{\delta \leq x<\infty \\ 0 \leq t \leq T}}\left|u^{\varepsilon}(x, t)\right|=0
$$

for each $\delta>0$.

Notice that in the first example $u^{\varepsilon}(x, t)$ preserves the boundary condition and in the second example $u^{\varepsilon}(x, t)$ loses the boundary condition as $\varepsilon \rightarrow 0$. Now we consider the general case.

\section{RESULT FOR LINEAR SYSTEMS}

Let $u=\left(u_{1}, \ldots, u_{m}\right) \in R^{m}$. We use the following notations:

$$
\begin{aligned}
& (u)_{k}=u_{k}, \\
& \|u\|=\left(\int_{0}^{1}|u|^{2} d x\right)^{1 / 2} .
\end{aligned}
$$

Let $u^{\varepsilon}(x, t)$ be the solution of (1.2), (1.3), (1.4) and (1.5), and let $u^{0}(x, t)$ be the solution of (1.1) with initial and boundary conditions

$$
\begin{gathered}
u^{0}(x, t)=u_{0}(x), \\
l_{j}(0, t) u^{0}(0, t)=l_{j}(0, t) u_{1}(t), \quad j=k+1, \ldots, m, \\
l_{j}(1, t) u^{0}(1, t)=l_{j}(1, t) u_{2}(t), \quad j=1,2, \ldots, k .
\end{gathered}
$$

Let $B=B(x, t, \varepsilon)$ be a function with the following properties,

$$
\begin{gathered}
B(0, t, \varepsilon)=u_{1}(t)-u^{0}(0, t), \\
B(1, t, \varepsilon)=u_{2}(t)-u^{0}(1, t), \\
B(x, t, \varepsilon)=T(x, t)\left\{\left[p_{0}\left(\frac{x}{\varepsilon}, t\right)+\varepsilon p_{1}\left(\frac{x}{\varepsilon}, t\right)\right] \phi_{1}(x)\right. \\
\left.+\left[q_{0}\left(\frac{x-1}{\varepsilon}, t\right)+\varepsilon q_{1}\left(\frac{x-1}{\varepsilon}, t\right)\right] \phi_{2}(x)\right\}
\end{gathered}
$$


where $T(x, t)=\left(r_{1}(x, t), \ldots, r_{m}(x, t)\right)$, the matrix whose $j$ th column is the right eigenvector of $A$ corresponding to $\lambda_{j}$.

$$
p_{0}(y, t)=M_{0}(t)\left|\begin{array}{c}
e^{\lambda_{1}(0, t) y} \\
\vdots \\
e^{\lambda_{k}(0, t) y} \\
0 \\
\vdots \\
0
\end{array}\right|
$$

$(2.5)_{0}$

$$
\begin{aligned}
p_{1}(y, t)=c_{1}(t)+M_{1}(t)\left|\begin{array}{c}
e^{\lambda_{1}(0, t) y} \\
\vdots \\
e^{\lambda_{k}(0, t) y} \\
0 \\
\vdots \\
0
\end{array}\right| \\
+y M_{2}(t)\left|\begin{array}{c}
e^{\lambda_{1}(0, t) y} \\
\vdots \\
e^{\lambda_{k}(0, t) y} \\
0 \\
\vdots \\
0
\end{array}\right|+y^{2} M_{3}(t)\left|\begin{array}{c}
e^{\lambda_{1}(0, t) y} \\
\vdots \\
e^{\lambda_{k}(0, t) y} \\
0 \\
\vdots \\
0
\end{array}\right|,
\end{aligned}
$$

$$
q_{0}(y, t)=N_{0}(t)\left|\begin{array}{c}
0 \\
\vdots \\
0 \\
e^{\lambda_{k+1}(1, t) y} \\
\vdots \\
e^{\lambda_{m}(1, t) y}
\end{array}\right|
$$

$$
q_{1}(y, t)=d_{1}(t)+N_{1}(t)\left|\begin{array}{c}
0 \\
\vdots \\
0 \\
e^{\lambda_{k+1}(1, t) y} \\
\vdots \\
e^{\lambda_{m}(1, t) y}
\end{array}\right|
$$

$$
+y N_{2}(t)\left|\begin{array}{c}
0 \\
\vdots \\
0 \\
e^{\lambda_{k+1}(1, t) y} \\
\vdots \\
e^{\lambda_{m}(1, t) y}
\end{array}\right|+y^{2} N_{3}(t)\left|\begin{array}{c}
0 \\
\vdots \\
0 \\
e^{\lambda_{k+1}(1, t) y} \\
\vdots \\
e^{\lambda_{m}(1, t) y}
\end{array}\right| .
$$

$c_{1}(t)$ and $d_{1}(t)$ are smooth vectors, and $M_{i}(t)$ and $N_{i}(t)$ are smooth matrices which depend only on $t$ and are constructed in the proof of Lemma $1 . \phi_{1}(x)$ 
and $\phi_{2}(x)$ are $C^{\infty}$ cut off functions in $[0,1]$ :

$$
\begin{aligned}
& \phi_{1}(x)= \begin{cases}1 & \text { in } 0 \leq x \leq \delta / 2 \\
0 & \text { in } \delta \leq x \leq 1\end{cases} \\
& \phi_{2}(x)= \begin{cases}0 & \text { in } 0 \leq x \leq 1-\delta \\
1 & \text { in } 1-\delta / 2 \leq x \leq 1\end{cases}
\end{aligned}
$$

We prove the following

Theorem.

$$
\sup _{\substack{0 \leq x \leq 1 \\ 0 \leq t \leq T}}\left|u^{\varepsilon}(x, t)-u^{0}(x, t)-B(x, t, \varepsilon)\right| \leq c \varepsilon^{1 / 4}
$$

where $c$ depends only on $T$.

Remark. From the above theorem it follows that $u^{\varepsilon}(x, t) \rightarrow u^{0}(x, t)$ uniformly in $D_{\delta^{\prime}, T}=\left\{\left(x, t: \delta^{\prime} \leq x \leq 1-\delta^{\prime}, 0 \leq t \leq T\right\}\right.$ for each $\delta^{\prime}>0, T>0$, and in the limit $u^{\varepsilon}(x, t)$ preserves the boundary condition at $x=0, P^{+} u_{1}(t)$ and at $x=1, P^{-} u_{2}(t)$.

The proof of this theorem follows from two lemmas. Denote by

$$
T(x, t)=\left(r_{1}(x, t), \ldots, r_{m}(x, t)\right)
$$

the matrix whose $j$ th column is $r_{j}(x, t)$, the right eigenvalue of $A$. Then

$$
A(x, t) T(x, t)=T(x, t) D(x, t)
$$

where

$$
D(x, t)=\operatorname{diag}\left(\lambda_{1}(x, t), \ldots, \lambda_{m}(x, t)\right)
$$

is the diagonal matrix whose $j$ th diagonal entry is $\lambda_{j}(x, t)$. Set

$$
\begin{gathered}
u=T v, \quad u_{t}=T v+T v_{t}, \quad u_{x}=T_{x} v+T v_{x}, \\
u_{x x}=T_{x x} v+2 T_{x} v_{x}+T v_{x x},
\end{gathered}
$$

so that from (1.2) we get $v_{t}=L_{\varepsilon} v$, where

$$
\begin{aligned}
& L_{\varepsilon} v=\varepsilon v_{x x}+(\varepsilon P-D) v_{x}+(R+\varepsilon Q) v, \\
& P=P(x, t)=2 T^{-1} T_{x}, \\
& Q=Q(x, t)=T^{-1} T_{x x}, \\
& R=R(x, t)=-T^{-1} A T_{x}-T^{-1} T_{t}
\end{aligned}
$$

Denote

$$
v_{1}(t)=T^{-1}(0, t) u_{1}(t), \quad v_{2}(t)=T^{-1}(1, t) u_{2}(t), \quad v_{0}(x)=T^{-1}(x, 0) u_{0}(x)
$$

Define $v^{\varepsilon}=T^{-1} u^{\varepsilon}$; then for $v=v^{\varepsilon}$ the problem becomes

$$
\begin{gathered}
v_{t}^{\varepsilon}=L_{\varepsilon} v^{\varepsilon} \\
v^{\varepsilon}(x, 0)=v_{0}(x), \\
v^{\varepsilon}(0, t)=v_{1}(t), \\
v^{\varepsilon}(1, t)=v_{2}(t)
\end{gathered}
$$


The problem for $u^{0}$, in $v$ coordinates, becomes

$$
\begin{gathered}
v_{t}^{0}=L_{0} v^{0}, \\
v^{0}(x, 0)=v_{0}(x), \\
\left(v^{0}(0, t)\right)_{j}=\left(v_{1}(t)\right)_{j}, \quad j=k+1, \ldots, m, \\
\left(v^{0}(1, t)\right)_{j}=\left(v_{2}(t)\right)_{j}, \quad j=1,2, \ldots, k .
\end{gathered}
$$

We first prove the following

Lemma 1. There exist functions $b^{\varepsilon}(x, t), g(x, t, \varepsilon)$ and $k(x, \varepsilon)$ such that

$$
b_{t}^{\varepsilon}=L_{\varepsilon} b^{\varepsilon}+\varepsilon g(x, t, \varepsilon) \text {. }
$$

$b^{\varepsilon}(x, t)$ is of the form

$$
\begin{aligned}
b^{\varepsilon}(x, t)= & {\left[p_{0}(x / \varepsilon, t)+\varepsilon p_{1}(x / \varepsilon, t)\right] \phi_{1}(x) } \\
& +\left[q_{0}((x-1) / \varepsilon, t)+\varepsilon q_{1}((x-1) / \varepsilon, t)\right] \phi_{2}(x),
\end{aligned}
$$

where $p_{0}, p_{1}, \varepsilon_{0}, q, \phi_{1}$ and $\phi_{2}$ are as in $(2.4)_{0},(2.4)_{1},(2.5)_{0},(2.5)$, and $(2.5)_{3}$ respectively.

$$
\begin{aligned}
& b^{\varepsilon}(0, t)=v^{\varepsilon}(0, t)-v^{0}(0, t), \\
& b^{\varepsilon}(1, t)=v^{\varepsilon}(1, t)-v^{0}(1, t), \\
& b^{\varepsilon}(x, 0)=\varepsilon k(x, \varepsilon) .
\end{aligned}
$$

With $c$ depending only on $T$,

$$
\begin{gathered}
\sup _{0 \leq t \leq T}\|g(x, t, \varepsilon)\| \leq c, \\
\sup _{0 \leq t \leq T}\left\|g_{t}(x, t, \varepsilon)\right\| \leq c, \\
\left\|\partial_{x}^{k} k(x, \varepsilon)\right\|^{2} \leq c \varepsilon^{1-2 k}, \quad k=1,2, \\
\|k(x, \varepsilon)\|^{2} \leq c .
\end{gathered}
$$

Proof. Expanding the coefficient of the differential operator $L_{\varepsilon}$ in powers of $x$ near $x=0$, we get

$$
\begin{aligned}
& P(x, t)=P(0, t)+x P_{1}(x, t), \\
& R(x, t)=R(0, t)+x R_{1}(x, t), \\
& \lambda_{i}(x, t)=\lambda_{i}(0, t)+x \lambda_{i}^{\prime}(0, t)+x^{2} \lambda_{i 1}(x, t),
\end{aligned}
$$

where $P_{1}(x, t)$ and $R_{1}(x, t)$ are smooth matrices, and $\lambda_{i 1}$ is a smooth function. Set

$$
\frac{x}{\varepsilon}=y, \quad \partial_{x}=\frac{1}{\varepsilon} \partial_{y} .
$$

We seek $b^{\varepsilon}(x, t)$ near $x=0$ in the form

$$
b^{\varepsilon}(x, t)=p_{0}(x / \varepsilon, t)+\varepsilon p_{1}(x / \varepsilon, t)=p(x / \varepsilon, t) .
$$


In the $y$ variable we get from (2.2), (2.6) and (2.7),

$$
\begin{aligned}
\left(L_{\varepsilon}-\partial_{t}\right) p(y, t)= & \frac{\varepsilon}{\varepsilon^{2}} p_{y y}+\frac{\varepsilon}{\varepsilon}\left[P(0, t)+\varepsilon y P_{1}(\varepsilon y, t)\right] p_{y} \\
& -\frac{1}{\varepsilon}\left[D(0, t)+\varepsilon y D^{\prime}(0, t)+\varepsilon^{2} y^{2} D_{1}(\varepsilon y, t)\right] p_{y} \\
& +\varepsilon Q(\varepsilon y, t) p+\left[R(0, t)+\varepsilon y R_{1}(\varepsilon y, t)\right] p-p_{t}
\end{aligned}
$$

where

$$
D^{\prime}(0, t)=\operatorname{diag}\left[\partial_{x} \lambda_{1}(x, t), \ldots, \partial_{x} \lambda_{m}(x, t)\right] \text { at } x=0,
$$

and $D_{1}(z, t)$ is a smooth diagonal matrix. Rewriting (2.19), we get

$$
\left(L_{\varepsilon}-\partial_{t}\right) p=\left(\frac{1}{\varepsilon} L_{1}+L_{2}+\varepsilon L_{3}\right) p
$$

where

$$
\begin{aligned}
& L_{1} p=p_{y y}-D(0, t) p_{y}, \\
& \begin{aligned}
L_{2} p=\left[P(0, t)-y D^{\prime}(0, t)\right] p_{y}+R(0, t) p-\partial_{t} p \\
L_{3} p=y p_{1}(\varepsilon y, t) p_{y}-y^{2} D_{1}(\varepsilon y, t) p_{y}+\left[Q(\varepsilon y, t)+y R_{1}(\varepsilon y, t)\right] p \\
\left(L_{\varepsilon}-\partial_{t}\right) p=\left(\frac{1}{\varepsilon} L_{1}+L_{2}+\varepsilon L_{3}\right)\left\{p_{0}(y, t)+\varepsilon p_{1}(y, t)\right\} \\
=\frac{1}{\varepsilon} L_{1} p_{0}(y, t)+L_{1} p_{1}(y, t)+L_{2} p_{0}(y, t) \\
\quad+\varepsilon\left[L_{2} p_{1}+L_{3} p_{0}\right]+\varepsilon^{2} L_{3} p_{1}
\end{aligned}
\end{aligned}
$$

we choose $p_{0}$ such that

(2.22) $L_{1} p_{0}=0, \quad p_{0}(0, t)=\left(v_{1}(0, t)-v^{0}(0, t)\right), \quad p_{0}(y, t) \rightarrow 0 \quad$ as $y \rightarrow \infty$.

Notice that by $(2.10)$ and $(2.10)^{\prime}$

$$
\left(p_{0}(0, t)\right)_{j}=0 \text { for } j=k+1, \ldots, m .
$$

Since $\left(p_{0}(y, t)\right)_{j}$ has to satisfy (2.22) we have

$$
\begin{aligned}
& \partial_{y}^{2}\left(p_{0}(y, t)\right)_{j}=\lambda_{j}(0, t) \partial_{y}\left(p_{0}(y, t)\right)_{j}, \\
&\left(p_{0}(0, t)\right)_{j}= \begin{cases}0, & \text { for } j=k+1, \ldots, m, \\
\left(v_{1}(0, t)-v^{0}(0, t)\right)_{j}, & \text { for } j=0,1, \ldots, k .\end{cases}
\end{aligned}
$$

So we take

$$
\begin{aligned}
\left(p_{0}(y, t)\right)=\left(\left(v_{1}(0, t)-v^{0}(0, t)\right) e^{\lambda_{1}(0, t) y}, \ldots,\right. \\
\left.\left(v_{1}(0, t)-v^{0}(0, t)\right)_{k} e^{\lambda_{k}(0, t) y}, 0, \ldots, 0\right)^{t} .
\end{aligned}
$$

Now choose $p_{1}$ such that

$$
L_{1} p_{1}=-L_{2} p_{0}, \quad p_{1}(0, t)=0, \quad p(y, t) \rightarrow 0 \quad \text { as } y \rightarrow \infty,
$$


i.e.

$$
\begin{aligned}
& \partial_{y}^{2}\left(p_{1}\right)_{j}=\lambda_{j}(0, t) \partial_{y}\left(p_{1}\right)_{j}-\left(L_{2} p_{0}\right)_{j} \\
& \left(p_{1}\right)_{j}(0, t)=0, \quad(p)_{j} \rightarrow 0 \quad \text { as } y \rightarrow \infty
\end{aligned}
$$

By variation of the constant formula, the solution of $(2.24)_{1}$ and $(2.24)_{2}$ is

$$
\begin{aligned}
\left(p_{1}\right)_{j}(y, t)= & c_{j}(t)\left[1-e^{\lambda_{j}(0, t) y}\right] \\
& -\frac{1}{\lambda_{j}(0, t)} \int_{0}^{y}\left(e_{1}^{\lambda_{j}(0, t)\left(y-y^{\prime}\right)}\right)\left(L_{2} p_{0}\right)_{j} d y
\end{aligned}
$$

$c_{j}(t)$ is to be chosen properly so that $\left(p_{1}\right)_{j}(y, t) \rightarrow 0$ as $y \rightarrow \infty$.

Now

$$
\begin{aligned}
L_{2} p_{0}= & \alpha(t)\left(e^{\lambda_{1}(0, t) y}, \ldots, e^{\lambda_{k}(0, t) y}, 0 \ldots, 0\right) \\
& +y \beta(t)\left(e^{\lambda_{1}(0, t) y}, \ldots, e^{\lambda_{k}(0, t) y}, 0, \ldots, 0\right)
\end{aligned}
$$

where

$$
\begin{aligned}
& \alpha(t)=[P(0, t) D(0, t)+R(0, t)] \operatorname{diag}\left[p_{0}(0, t)\right]-\operatorname{diag}\left[\partial_{t} p_{0}(0, t)\right], \\
& \beta(t)=-D^{\prime}(0, t)[I+D(0, t)] \operatorname{diag}\left[p_{0}(0, t)\right] .
\end{aligned}
$$

Let us use the notation $\alpha(t)=\left(\alpha_{i j}(t)\right)$ and $\beta(t)=\left(\beta_{i j}(t)\right)$. From (22.6),

$$
\begin{aligned}
\int_{0}^{y}\left(e^{\lambda_{j}(0, t)\left(y-y^{\prime}\right)}-1\right)\left(L_{2} p_{0}\right)_{j} d y^{\prime} \\
=\int_{0}^{y}\left(e^{\lambda_{j}(0, t)\left(y-y^{\prime}\right)}-1\right) \sum_{i=1}^{k} \alpha_{i j}(t) e^{\lambda_{i}(0, t) y^{\prime}} d y^{\prime} \\
\quad+\int_{0}^{y}\left(e^{\lambda_{j}(0, t)\left(y-y^{\prime}\right)}-1\right) \sum_{i=1}^{k} \beta_{i j}(t) e^{\lambda_{i}(0, t) y^{\prime}} y^{\prime} d y^{\prime} \\
=I_{1}^{j}+I_{2}^{j} .
\end{aligned}
$$

We first consider

$$
\begin{aligned}
I_{1}^{j}= & e^{\lambda_{j}(0, t) y} \sum_{i=1}^{k} \alpha_{i j}(t) \int_{0}^{y} e^{\left(\lambda_{i}-\lambda_{j}\right) y^{\prime}} d y^{\prime} \\
& -\sum_{i=1}^{k} \alpha_{i j}(t) \int_{0}^{y} e^{\lambda_{i}(0, t) y^{\prime}} d y^{\prime}
\end{aligned}
$$

Rewriting this, we obtain

$$
\begin{aligned}
I_{1}^{j}= & e^{\lambda_{j}(0, t) y} \sum_{\substack{i=1 \\
j \neq i}}^{k} \alpha_{i j}(t) \int_{0}^{y} e^{\left(\lambda_{i}-\lambda_{j}\right) y^{\prime}} d y^{\prime} \\
& +e^{\lambda_{j} y} \alpha_{j j}(t) y-\sum_{i=1}^{k} \frac{\alpha_{i j}(t)}{\lambda_{i}(0, t)}\left(e^{\lambda_{i}(0, t) y}-1\right) .
\end{aligned}
$$


For $i \neq j$,

$$
\int_{0}^{y} e^{\left(\lambda_{i}-\lambda_{j}\right) y^{\prime}} d y^{\prime}=\frac{1}{\lambda_{i}-\lambda_{j}}\left(e^{\left(\lambda_{i}-\lambda_{j}\right) y}-1\right) .
$$

Substituting this in (2.27) we get

$$
\begin{aligned}
I_{1}^{j}= & \sum_{\substack{i=1 \\
j \neq i}}^{k} \frac{\alpha_{i j}(t)}{\left(\lambda_{i}(0, t)-\lambda_{j}(0, t)\right)}\left(e^{\lambda_{i}(0, t) y}-e^{\lambda_{j}(0, t) y}\right) \\
& -\sum_{i=1}^{k} \frac{\alpha_{i j}(t)}{\lambda_{i}(0, t)}\left(e^{\lambda_{i}(0, t) y}-1\right)+\alpha_{j j}(t) y e^{\lambda_{j}(0, t) y} .
\end{aligned}
$$

Now we consider $I_{2}^{j}$, where

$$
\begin{aligned}
I_{2}^{j}= & e^{\lambda_{j}(0, t) y} \sum_{i=1}^{k} \beta_{i j}(t) \int_{0}^{y} e^{\left(\lambda_{i}(0, t)-\lambda_{j}(0, t)\right) y^{\prime}} y^{\prime} d y^{\prime} \\
& -\sum_{i=1}^{k} \beta_{i j}(t) \int_{0}^{y} y^{\prime} e^{\lambda_{i}(0, t) y^{\prime}} d y^{\prime} .
\end{aligned}
$$

\section{Rewriting this we get}

$$
\begin{aligned}
I_{2}^{j}= & e^{\lambda_{j}(0, t) y} \sum_{\substack{i=1 \\
i \neq j}}^{k} \beta_{i j}(t) \int_{0}^{y} e^{\left(\lambda_{i}(0, t)-\lambda_{j}(0, t)\right) y^{\prime}} y^{\prime} d y^{\prime} \\
& +e^{\lambda_{j}(0, t) y} \beta_{j j}(t) \frac{y^{2}}{2}-\sum_{i=1}^{k} \beta_{i j}(t) \int_{0}^{y} y^{\prime} e^{\lambda_{i}(0, t) y^{\prime}} d y^{\prime} .
\end{aligned}
$$

For $i \neq j$,

$$
\begin{gathered}
\int_{0}^{y} y^{\prime} e^{\left(\lambda_{i}(0, t)-\lambda_{j}(0, t)\right) y^{\prime}} d y^{\prime} \\
=\left.\frac{y^{\prime} e^{\left(\lambda_{i}(0, t)-\lambda_{j}(0, t)\right) y^{\prime}}}{\left(\lambda_{i}(0, t)-\lambda_{j}(0, t)\right)}\right|_{0} ^{y}-\int_{0}^{y} \frac{e^{\left(\lambda_{i}(0, t)-\lambda_{j}(0, t)\right) y^{\prime}}}{\left(\lambda_{i}(0, t)-\lambda_{j}(0, t)\right)} d y^{\prime} \\
=\frac{y e^{\left(\lambda_{i}(0, t)-\lambda_{j}(0, t)\right) y}}{\left(\lambda_{i}(0, t)-\lambda_{j}(0, t)\right)}-\frac{\left(e^{\left(\lambda_{i}(0, t)-\lambda_{j}(0, t)\right) y}-1\right)}{\left(\lambda_{i}(0, t)-\lambda_{j}(0, t)\right)^{2}}, \\
\int_{0}^{y} y^{\prime} e^{\lambda_{i}(0, t) y^{\prime}} d y^{\prime}=\left.\frac{y^{\prime}}{\lambda_{i}(0, t)} e^{\lambda_{i}(0, t) y^{\prime}}\right|_{0} ^{y}-\frac{1}{\lambda_{i}(0, t)} \int_{0}^{y} e^{\lambda_{i}(0, t) y^{\prime}} d y^{\prime} \\
=\frac{y}{\lambda_{i}(0, t)} e^{\lambda_{i}(0, t) y}-\frac{1}{\lambda_{i}(0, t)^{2}}\left(e^{\lambda_{i}(0, t) y}-1\right) .
\end{gathered}
$$


Substituting these in (2.29), we obtain

$$
\begin{aligned}
I_{2}^{j}= & \frac{y^{2}}{2} \beta_{j j}(t) e^{\lambda_{j} y}+y\left[\sum_{\substack{i=1 \\
i \neq j}} \frac{\beta_{i j}(t)}{\left(\lambda_{i}(0, t)-\lambda_{j}(0, t)\right)}-\sum_{i=1}^{k} \frac{\beta_{i j}(t)}{\lambda_{i}(0, t)}\right] e^{\lambda_{i}(0, t) y} \\
& +\sum_{i=1}^{k} \frac{\beta_{i j}(t) e^{\lambda_{i} y}}{\lambda_{i}(0, t)}-\sum_{\substack{i=1 \\
i \neq j}}^{k} \frac{\beta_{i j}(t) e^{\lambda_{i} y}}{\left(\lambda_{i}-\lambda_{j}\right)^{2}} \\
& +\left(\sum_{\substack{i=1 \\
i \neq j}}^{k} \frac{\beta_{i j}}{\left(\lambda_{i}-\lambda_{j}\right)^{2}}\right) e^{\lambda_{j} y}-\sum_{i=1}^{k} \frac{\beta_{i j}(t)}{\lambda_{i}^{2}(0, t)} .
\end{aligned}
$$

From (2.28), (2.30) and (2.25) we get, for $j=1,2, \ldots, k$,

$$
\begin{aligned}
\left(p_{1}\right)_{j}=c_{j}(t)\left[1-e^{\lambda_{j}(0, t) y}\right] & \\
-\frac{1}{\lambda_{j}(0, t)}[ & \sum_{i=1}^{k} \frac{\alpha_{i j}(t)\left(e^{\lambda_{i}(0, t) y}-e^{\left.\lambda_{j}(0, t) y\right)}\right.}{\left(\lambda_{i}(0, t)-\lambda_{j}(0, t)\right)} \\
& \left.+\alpha_{j j}(t) y e^{\lambda_{j}(0, t) y}-\sum_{i=1}^{k} \frac{\alpha_{i j}(t)}{\lambda_{i}(0, t)}\left(e^{\lambda_{i}(0, t) y}-1\right)\right] \\
-\frac{1}{\lambda_{j}(0, t)}[ & \frac{y^{2}}{2} \beta_{j j}(t) e^{\lambda_{j}(0, t) y} \\
& +y\left(\sum_{\substack{i=1 \\
i \neq j}}^{k} \frac{\beta_{i j}(t) e^{\lambda_{i}(0, t) y}}{\left(\lambda_{i}(0, t)-\lambda_{j}(0, t)\right)}-\sum_{i=1}^{k} \frac{\beta_{i j}(t) e^{\lambda_{i}(0, t) y}}{\lambda_{i}(0, t)}\right) \\
& +\sum_{i=1}^{k} \frac{\beta_{i j}(t) e^{\lambda_{i} y}}{\lambda_{i}^{2}(0, t)}-\sum_{\substack{i=1 \\
i \neq j}}^{k} \frac{\beta_{i j}(t) e^{\lambda_{i} y}}{\left(\lambda_{i}(0, t)-\lambda_{j}(0, t)\right)^{2}} \\
& \left.+\left(\sum_{\substack{i=1 \\
i \neq j}}^{k} \frac{\beta_{i j}(t)}{\lambda_{i}(0, t)-\lambda_{j}(0, t)}\right) e^{\lambda_{j}(0, t) y}-\sum_{i=1}^{k} \frac{\beta_{i j}(t)}{\lambda_{i}^{2}(0, t)}\right] .
\end{aligned}
$$

The choice of $c_{j}(t)$. 
Case $j=1,2, \ldots, k$.

Since $p_{0}(0,0)=0$ from equation $(2.26)^{\prime}$ we have $\beta_{i j}(0)=0$ and $\alpha_{i j}(0)=$ $\operatorname{diag}\left(\partial_{t}(p(0,0))\right)$. From $(2.31)$, we get

$$
\begin{aligned}
& \left(p_{1}\right)_{j}(y, 0)=c_{j}(0)\left[1-e^{\lambda_{j}(0,0) y}\right] \\
& -\frac{1}{\lambda_{j}(0,0)}\left[\sum_{\substack{i=1 \\
i \neq j}}^{k} \frac{\alpha_{i j}(0)}{\lambda_{i}(0,0)-\lambda_{j}(0,0)}\left(e^{\lambda_{i}(0,0) y}-e^{\lambda_{j}(0,0) y}\right)\right. \\
& \\
& \left.\quad+\alpha_{j j}(0) y e^{\lambda_{j}(0,0) y}-\sum_{i=1}^{k} \frac{\alpha_{i j}(0)}{\lambda_{i}(0,0)}\left(e^{\lambda_{i}(0,0) y}-1\right)\right] .
\end{aligned}
$$

Take $c_{j}(t)=1$.

Case $j=1+1, \ldots, m$.

In this case in the formulas for $I_{1}^{j}$ and $I_{2}^{j}$ the summation in $i$ is only from 1 to $k$, and for $i=1,2, \ldots, k, i \neq j$,

$$
\begin{aligned}
& \left(p_{1}\right)_{j}=c_{j}(t)\left[1-e^{\lambda_{j}(0, t) y}\right] \\
& -\frac{1}{\lambda_{j}(0, t)}\left\lceil\sum_{i=1}^{k} \frac{\alpha_{i j}(t)\left(e^{\lambda_{i}(0, t) y}-e^{\lambda_{j}(0, t) y}\right)}{\left(\lambda_{i}(0, t)-\lambda_{j}(0, t)\right)}\right. \\
& -\sum_{i=1}^{k} \frac{\alpha_{i j}(t)}{\lambda_{i}(0, t)}\left(e^{\lambda_{i}(0, t) y}-1\right) \\
& -\frac{y}{\lambda_{j}(0, t)}\left[\sum_{i=1}^{k} \frac{\beta_{i j}(t) e^{\lambda_{i}(0, t) y}}{\left(\lambda_{i}(0, t)-\lambda_{j}(0, t)\right)}-\sum_{i=1}^{k} \frac{\beta_{i j}(t) e^{\lambda_{i}(0, t) y}}{\lambda_{i}(0, t)}\right] \\
& -\frac{1}{\lambda_{j}(0, t)}\left[\sum_{i=1}^{k} \frac{\beta_{i j}(t) e^{\lambda_{i}(0, t) y}}{\lambda_{i}^{2}(0, t)}-\sum_{i=1}^{k} \frac{\beta_{i j}(t) e^{\lambda_{i}(0, t) y}}{\left(\lambda_{i}(0, t)-\lambda_{j}(0, t)\right)^{2}}\right. \\
& \left.+\left(\sum_{i=1}^{k} \frac{\beta_{i j}(t)}{\lambda_{i}(0, t)-\lambda_{j}(0, t)}\right) e^{\lambda_{j}(0, t) y}-\sum_{i=1}^{k} \frac{\beta_{i j}(t)}{\lambda_{i}^{2}(0, t)}\right] .
\end{aligned}
$$

Since $\lambda_{j}(0, t)>0, j=k+1, \ldots, m$, the $e^{\lambda_{j}(0, t) y}$ are bad terms, so we choose $c_{j}(t)$ such that

$$
\begin{aligned}
-c_{j}(t)+\frac{1}{\lambda_{j}(0, t)}\left[\sum_{i=1}^{k}\right. & \frac{\alpha_{i j}(t)}{\lambda_{i}(0, t)-\lambda_{j}(0, t)} \\
& \left.-\sum_{i=1}^{k} \frac{\beta_{i j}(t)}{\left(\lambda_{i}(0, t)-\lambda_{j}(0, t)\right)}\right]=0 .
\end{aligned}
$$


We get from (2.33) and (2.34), for $j=k+1, \ldots, m$,

$$
\begin{aligned}
\left(p_{1}\right)_{j}(y, t)= & c_{j}(t)-\frac{1}{\lambda_{j}(0, t)}\left[\sum_{i=1}^{k} \frac{\alpha_{i j}(t) e^{\lambda_{i}(0, t) y}}{\left(\lambda_{i}(0, t)-\lambda_{j}(0, t)\right)}\right. \\
& -\frac{y}{\lambda_{j}(0, t)}\left[\sum_{i=1}^{k} \frac{\alpha_{i j}(t)}{\lambda_{i}(0, t)}\left(e^{\lambda_{i}(0, t) y}-1\right)\right] \\
& -\frac{1}{\lambda_{j}(0, t)}\left[\sum_{i=1}^{k} \frac{\beta_{i j}(t) e^{\lambda_{i}(0, t) y}}{\left.\lambda_{i}(0, t)-\lambda_{j}(0, t)\right)}-\sum_{i=1}^{k} \frac{\beta_{i j}(t) e^{\lambda_{i}(0, t) y}}{\lambda_{i}^{2}(0, t)}\right] \\
& \left.-\sum_{i=1}^{k} \frac{\beta_{i j}(t) e^{\lambda_{i}(0, t) y}}{\left(\lambda_{i}(0, t)-\lambda_{j}(0, t)\right)}-\sum_{i=1}^{k} \frac{\beta_{i j}(t)}{\lambda_{i}^{2}(0, t)}\right],
\end{aligned}
$$

where $c_{j}(t)$ is given by $(2.34)$. For $j=k+1, \ldots, m$,

$$
\begin{aligned}
\left(p_{1}\right)_{j}(y, 0)=c_{j}(0)-\frac{1}{\lambda_{j}(0,0)}[ & {\left[\sum_{i=1}^{k} \frac{\alpha_{i j}(0) e^{\lambda_{i}(0,0) y}}{\left(\lambda_{i}(0,0)-\lambda_{j}(0,0)\right)}\right.} \\
& \left.-\sum_{i=1}^{k} \frac{\alpha_{i j}(0,0)}{\lambda_{i}(0,0)}\left(e^{\lambda_{i}(0,0) y}-1\right)\right] .
\end{aligned}
$$

Now let $\phi_{1}(x)$ be a $C^{\infty}$ function in $[0,1]$ such that

$$
\begin{array}{ll}
\phi_{1}(x)=1 & \text { in } 0 \leq x \leq \delta / 2 \\
\phi_{1}(x)=0 & \text { in } \delta \leq x \leq 1
\end{array}
$$

Set

$$
b_{1}^{\varepsilon}(x, t)=\left[p_{0}(x / \varepsilon, t)+\varepsilon p_{1}(x / \varepsilon, t)\right] \phi_{1}(x) .
$$

$b_{1}^{\varepsilon}(x, t)$ is the boundary layer function near $x=0$ with the following important properties.

(a) $\left(p_{0}(y)\right)_{j}$ is a linear combination of $e^{\lambda_{1}(0, t) y}, \ldots, e^{\lambda_{k}(0, t) y}$ whose coefficients depend smoothly on $t$ and on nothing else.

(b) $\left(p_{1}(y)\right)_{j}$ is a linear combination of $e^{\lambda_{t}(0, t)}, y e^{\lambda_{t}(0, t)}$ and $y^{2} e^{\lambda_{1}(0, t)}, i=1,2, \ldots, k$, whose coefficients depend smoothly on $t$ and on nothing else.

(c) $p_{0}(0, t)=v_{1}(0, t)-v^{0}(0, t), p_{1}(0, t)=0$.

(d) $p_{0}(y, 0)=0, p_{1}(y, 0)$ is a linear combination of $1, e^{\lambda_{l}(0.0) y}, y e^{\lambda_{l}(1.0) y}, i=1,2, \ldots, k$, whose coefficients are constants. 
By a similar procedure, expanding the coefficients of $L_{\varepsilon}$ in powers of $(x-1)$, near $x=1$ we construct a boundary layer function $b_{2}^{\varepsilon}(x, t)$.

The function $b^{\varepsilon}(x, t)$ we are seeking is given by

$$
b^{\varepsilon}(x, t)=b_{1}^{\varepsilon}(x, t)+b_{2}^{\varepsilon}(x, t) .
$$

Define $k(x, \varepsilon)$ by

$$
b^{\varepsilon}(x, 0)=\varepsilon \phi_{1}(x / \varepsilon, 0)=\varepsilon k(x, \varepsilon),
$$

and $g(x, t, \varepsilon)$ by

$$
\partial_{t} b^{\varepsilon}-L_{\varepsilon} b^{\varepsilon}=\varepsilon g(x, t, \varepsilon) .
$$

Notice that from (2.37) (a), (b), (c) and (d), for $b_{1}^{\varepsilon}(x, t)$ and similar properties of $b_{2}^{\varepsilon}(x, t), b^{\varepsilon}(x, t)$ is exponentially decreasing to 0 as $\varepsilon \rightarrow 0$ in the interval $\delta / 2 \leq x \leq 1-\delta / 2$ and so properties (2.14), (2.15), and (2.16) are verified there.

We now verify these properties in $0 \leq x \leq \delta / 2$ and in $1-\delta / 2 \leq x \leq 1$. We do our analysis in $0 \leq x \leq \delta / 2$; the other case is similar.

By $(2.32)$ and $(2.36),(k(x, \varepsilon))_{j}$ is a linear combination of $1, e^{\lambda_{i}(0,0) x / \varepsilon}$, $(x / \varepsilon) e^{\lambda_{i}(0,0) x / \varepsilon}$, so that

$$
\int_{0}^{\delta}|k(x, \varepsilon)|^{2} \leq c, \quad \int_{0}^{\delta}\left|k_{x}(x, \varepsilon)\right|^{2} \leq \frac{c}{\varepsilon}, \quad \int_{0}^{\delta}\left|k_{x x}(x, \varepsilon)\right|^{2} \leq \frac{c}{\varepsilon^{3}} .
$$

We also obtain

$$
\int_{\delta}^{1-\delta}\left|\partial_{k}^{k} k(x, t)\right| \leq e^{-M / \varepsilon}, \quad M>0
$$

The same estimate as in $(2.38)$ holds in the interval $[1-\delta, 1]$, so that we get

$$
\left\|\partial_{x}^{k} k(x, \varepsilon)\right\|^{2} \leq c \varepsilon^{1-2 k}, \quad k=1,2,
$$

and

$$
\|k(x, \varepsilon)\|^{2} \leq c .
$$

Now verify properties (2.14) and (2.15) of $g(x, t, \varepsilon)$. Here again we need to argue only in $0 \leq x \leq \delta / 2$. In this interval $\phi_{1}(x)=1$, so that, by $(2.21)$

$$
\partial_{t} b^{\varepsilon}-L_{\varepsilon} b^{\varepsilon}=\varepsilon\left[L_{2} p_{1}+L_{3} p_{0}+\varepsilon L_{3} p_{1}\right] y=x / \varepsilon
$$

The terms in $g(x, t, \varepsilon)$ and in $g_{t}(x, t, \varepsilon)$ are linear combinations of

$$
1,\left(\frac{x}{\varepsilon}\right)^{n} e^{\lambda_{i}(0, t) x / \varepsilon}, \quad i=1,2, \ldots, k,
$$

with the coefficient independent of $\varepsilon$. It is clear that

$$
\sup _{0 \leq t \leq T}\|g(x, t, \varepsilon)\|_{L^{2}[0, \delta]}^{2} \text { and } \sup _{0 \leq t \leq T}\left\|g_{t}(x, t, \varepsilon)\right\|_{L^{2}[0, \delta]}
$$

are bounded by $c$. 
We use a similar argument for the intervals $[1-\delta / 2,1]$ and $[\delta / 2,1-\delta / 2]$, where the terms in $g(x, t, \varepsilon)$ and $g_{t}(x, t, \varepsilon)$ are bounded. Hence

$$
\sup _{0 \leq t \leq T}\left\|g(x, t, \varepsilon) \leq c, \quad \sup _{0 \leq t \leq T}\right\| g_{t}(x, t, \varepsilon) \| \leq c,
$$

The proof of Lemma 1 is complete.

Define

$$
z^{\varepsilon}=v^{\varepsilon}-v^{0}-b^{\varepsilon}
$$

All the constants depend only on $T$.

Lemma 2.

$$
\sup _{\substack{0 \leq x \leq 1 \\ 0 \leq t \leq T}}\left|z^{\varepsilon}(x, t)\right| \leq c \varepsilon^{1 / 4},
$$

where $c$ depends only on $T$.

Proof. Clearly, by Lemma 1, (2.13),

$$
\begin{gathered}
z(0, t)=0=z(1, t), \\
z(x, 0)=\varepsilon k(x, \varepsilon), \\
z_{t}=\varepsilon z_{x x}+[-D(x, t)+\varepsilon P] z_{x}+[R+\varepsilon Q] z-\varepsilon h,
\end{gathered}
$$

where $h=h(x, t, \varepsilon)=g(x, t, \varepsilon)+v_{x x}^{0}+P v_{x}^{0}+Q v^{0}$. By using Lemma 1, we have

$$
\sup _{0 \leq t \leq T}\|h(x, t, \varepsilon)\| \leq c, \quad \sup _{0 \leq t \leq T}\left\|h_{t}(x, t, \varepsilon)\right\| \leq c .
$$

Multiply (2.44) by $z$ and integrate by parts with respect to $x$. Then using (2.42) we get

$$
\begin{aligned}
\frac{1}{2} \frac{d}{d t}\|z\|^{2} \leq & -\varepsilon\left\|z_{x}\right\|^{2}+c\|z\|^{2}+\varepsilon c\|z\|\left\|z_{x}\right\| \\
& +c\|z\|^{2}+\varepsilon c\|z\|^{2}+\varepsilon\|z\|\|h\| .
\end{aligned}
$$

Using $2 a b \leq \delta a^{2}+b^{2} / \delta$ we get

$$
\frac{d}{d t}\|z\|^{2} \leq c\|z\|^{2}+\varepsilon^{2}\|h\| .
$$

By Gronwall's inequality we get

$$
\|z(t)\|^{2} \leq e^{c t}\|z(0)\|^{2}+\varepsilon^{2} \int_{0}^{t}\|h\|(s) e^{c(t-s)} d s .
$$

By Lemma 1, $z(x, 0)=\varepsilon k_{\varepsilon}(x)$ and $\left\|k_{\varepsilon}(x)\right\|^{2} \leq c$, so that, from (2.47) we get

$$
\sup _{0 \leq t \leq T}\|z(t)\|^{2} \leq c \varepsilon^{2}\left[1+\sup _{0 \leq t \leq T}\|h\|^{2}(s)\right] .
$$

Using (2.45), we get

$$
\sup _{0 \leq t \leq T}\|z(t)\|^{2} \leq c \varepsilon^{2}
$$


Next we show

$$
\sup _{0 \leq t \leq T}\left\|\partial_{t} z(x, t)\right\|^{2} \leq c \varepsilon .
$$

Differentiating (2.44) for $z$ with respect to $t$, we get

$$
\begin{aligned}
\left(z_{t}\right)_{t}= & \varepsilon\left(z_{t}\right)_{x x}+(-D+\varepsilon P)\left(z_{t}\right)_{x}+\left(-D_{t}+\varepsilon P_{t}\right) z_{x} \\
& +[R+\varepsilon Q] z_{t}+\left(\varepsilon Q_{t}+R_{t}\right) z-\varepsilon h_{t}(x, t, \varepsilon) .
\end{aligned}
$$

Multiply (2.49) by $z_{t}$ and integrate with respect to $x$. We get for $0 \leq t \leq T$,

$$
\begin{aligned}
\frac{1}{2} \frac{d}{d t}\left\|z_{t}\right\|^{2} \leq & -\varepsilon\left\|z_{t x}\right\|^{2}+c\left\|z_{t}\right\|^{2}+\varepsilon c\left\|z_{t}\right\|\left\|z_{t x}\right\| \\
& +\int_{0}^{1}\left\langle D_{t} z_{x}, z_{t}\right\rangle d x+\varepsilon \int_{0}^{1}\left\langle p_{t} z_{x}, z_{t}\right\rangle d x \\
& +c\left\|z_{t}\right\|^{2}+c\left\|z_{t}\right\|\|z\|+\varepsilon\left\|z_{t}\right\|\left\|h_{t}\right\|,
\end{aligned}
$$

$$
\begin{aligned}
& \int_{0}^{1}\left\langle D_{t} z_{x}, z_{t}\right\rangle d x=\sum_{i=1}^{m} \int_{0}^{1}\left(\lambda_{i}\right)_{t}\left(z_{i}\right)_{x}\left(z_{i}\right)_{t} d x, \\
& \int_{0}^{1}\left(\lambda_{i}\right)_{t}\left(z_{i}\right)\left(z_{i}\right)_{t} d x=\int_{0}^{1}\left(\lambda_{i}\right)_{t}\left(z_{i}\right)_{t}\left(z_{i}\right)_{x} d x \\
&=-\int_{0}^{1}\left[\left(\lambda_{i}\right)_{t}\left(z_{i}\right)_{t}\right]_{x} z_{i} d x \\
&=-\int_{0}^{1}\left(\lambda_{i}\right)_{t x}\left(z_{i}\right)_{t} z_{i}-\int_{0}^{1}\left(\lambda_{i}\right)_{t}\left(z_{i}\right)_{t x} z_{i} d x \\
& \leq c\left[\|z\|\left\|z_{t}\right\|+\|z\|\left\|z_{t x}\right\|\right] .
\end{aligned}
$$

We get from (2.51) and (2.52)

$$
\int_{0}^{1}\left\langle D_{t} z_{x}, z_{t}\right\rangle d x \leq c\left[\|z\|\left\|z_{t}\right\|+\|z\|\left\|z_{t x}\right\|\right] .
$$

Since $\|z\| \leq c \varepsilon$, by (2.48), this is

$$
\begin{aligned}
& \leq c\left[\varepsilon\left\|z_{t}\right\|+\varepsilon\left\|z_{t x}\right\|\right] \\
\int_{0}^{1}\left\langle p_{t} z_{x}, z_{t}\right\rangle d x & =\int_{0}^{t}\left\langle z_{x}, p^{t} z_{t}\right\rangle d x .
\end{aligned}
$$

Integrating by parts, we get this is

$$
\begin{aligned}
& =\int_{0}^{t}\left\langle z,\left(p^{t} z_{t}\right)_{x}\right\rangle d x \\
& \leq c\|z\|\left[\left\|z_{t x}\right\|+\left\|z_{t}\right\|\right] .
\end{aligned}
$$

Since $\|z\| \leq c \varepsilon$ by $(2.48)$ this is

$$
\begin{aligned}
& \leq c \varepsilon\left[\left\|z_{t^{x}}\right\|+\left\|z_{t}\right\|\right] \\
& \leq \frac{1}{10}\left\|z_{t x}\right\|^{2}+c \varepsilon^{2}+c \varepsilon\left\|z_{t}\right\|
\end{aligned}
$$


where we used $2 a b \leq a^{2} / \delta+\delta b^{2}$. Using (2.50), (2.53) and (2.54), we obtain

$$
\begin{aligned}
\frac{d}{d t}\left\|z_{t}\right\|^{2} \leq & -\varepsilon\left\|z_{t} x\right\|^{2}+c\left\|z_{t}\right\|^{2}+\varepsilon c\left\|z_{t}\right\|\left\|z_{t x}\right\| \\
& +c\left[\varepsilon\left\|z_{t}\right\|+\varepsilon\left\|z_{t^{*}}\right\|\right]+\frac{\varepsilon}{10}\left\|z_{t x}\right\|^{2}+c \varepsilon^{3}+c \varepsilon^{2}\left\|z_{t}\right\| \\
& +c\left\|z_{t}\right\|^{2}+c\left\|z_{t}\right\|\|z\|+\varepsilon\left\|z_{t}\right\|\left\|h_{t}\right\| .
\end{aligned}
$$

Using (2.55) and $\|z\| \leq c \varepsilon$, we get

$$
\begin{aligned}
\frac{d}{d t}\left\|z_{t}\right\|^{2} \leq & -\varepsilon\left\|z_{t x}\right\|^{2}+c\left\|z_{t}\right\|^{2} \\
& +c \varepsilon+\varepsilon^{2}\left\|h_{t}\right\|^{2}+\varepsilon\|\| z_{t^{x}} \|^{2} .
\end{aligned}
$$

Using (2.45), we get

$$
\frac{d}{d t}\left\|z_{t}\right\|^{2} \leq c\left[\left\|z_{t}\right\|^{2}+\varepsilon\right]
$$

Using Gronwall's inequality we get

$$
\left\|z_{t}(t)\right\|^{2} \leq c\left[\left\|z_{t}(0)\right\|+\varepsilon\right] .
$$

Using the partial differential equation $(2.44)$ we can express $z_{t}(x, 0)$ as

$$
z_{t}(x, 0)=\left.L_{\varepsilon} z(x, t)\right|_{t=0}-\varepsilon h(x, 0, \varepsilon) .
$$

Since $z(x, 0)=\varepsilon k(x, \varepsilon)$, by $(2.43)$

$$
\begin{aligned}
z_{t}(x, 0)= & \varepsilon^{2} \partial_{x}^{2} k(x, \varepsilon)+\varepsilon[-D(x, 0)+\varepsilon p(x, 0)] \partial_{x} k(x, \varepsilon) \\
& +\varepsilon[R(x, 0)+\varepsilon Q(x, 0)] k(x, \varepsilon)-\varepsilon h(x, 0, \varepsilon) .
\end{aligned}
$$

By Lemma 1, (2.16) we have

$$
\begin{gathered}
\left\|\partial_{x}^{k} k(x, \varepsilon)\right\|^{2} \leq \varepsilon^{1-2 k}, \quad k=1,2, \\
\|k(x, \varepsilon)\|^{2} \leq c
\end{gathered}
$$

We get from (2.57)

$$
\left\|z_{t}(x, 0)\right\|^{2} \leq c \varepsilon
$$

so that from (2.56) we get

$$
\sup _{0 \leq t \leq T}\left\|z_{t}(x)\right\|^{2} \leq c \varepsilon
$$

Next we show

$$
\sup _{0 \leq t \leq T}\left\|z_{x}(t)\right\|^{2} \leq c \varepsilon
$$

Multiply (2.44) by $z$ and integrate with respect to $x$ and integrate by parts. We get

$$
\begin{aligned}
\varepsilon\left\|z_{x}\right\|^{2} \leq & \|z\|\left\|z_{t}\right\|+c\|z\|^{2}+\varepsilon c\|z\|\left\|z_{x}\right\|+c\|z\|^{2} \\
& +\varepsilon c\|z\|^{2}+\varepsilon\|z\|\|h\| .
\end{aligned}
$$

Using previous estimates (2.48) for $\|z\|^{2},(2.58)$ for $\left\|z_{t}\right\|$ and (2.45) for $\|h\|$, we get

$$
\varepsilon\left\|z_{x}\right\|^{2} \leq c \varepsilon^{3} / 2+c \varepsilon^{2}+c \varepsilon^{2}\left\|z_{x}\right\|+c \varepsilon^{2}+c \varepsilon^{3}+c \varepsilon^{2} .
$$


Using $c \varepsilon^{2}\left\|z_{x}\right\| \leq \varepsilon^{2}\left[c^{2}+\left\|z_{x}\right\|^{2}\right]$ we get for small $\varepsilon$,

$$
\left(\varepsilon-\varepsilon^{2}\right)\left\|z_{x}\right\|^{2} \leq c \varepsilon^{3 / 2}
$$

from which we get $\left\|z_{x}\right\|^{2} \leq c \varepsilon^{1 / 2}$ so that

$$
\sup _{0 \leq t \leq T}\left\|z_{x}(t)\right\|^{2} \leq c \varepsilon^{1 / 2} \text {. }
$$

Since $z(0, t)=0$,

$$
z(x, t)=\int_{0}^{x} z_{y}(y, t) d y .
$$

By Schwarz's inequality, $|z(x, t)| \leq\left\|z_{x}\right\|(t) \leq c \varepsilon^{1 / 4}$, i.e.

$$
\sup _{\substack{0 \leq x \leq 1 \\ 0 \leq t \leq T}}|z(x, t)| \leq c \varepsilon^{1 / 4}
$$

This completes the proof of Lemma 2.

Now we shall prove the theorem.

Proof of theorem. Let $u^{\varepsilon}(x, t)$ be the solution of (1.2), (1.3), (1.4) and (1.5) and $u^{0}(x, t)$ be the solution of $(1.1),(1.3)^{\prime},(1.4)^{\prime}$ and $(1.5)^{\prime}$. Since $u=T v$ by $(2.6)$, we get

$$
u^{\varepsilon}(x, t)=T(x, t) v^{\varepsilon}, \quad u^{0}(x, t)=T(x, t) v^{0}(x, t) .
$$

Denote $B(x, t, \varepsilon)=T(x, t) b^{\varepsilon}(x, t)$, where $b^{\varepsilon}(x, t)$ is given by Lemma 1 .

Consider

$$
\begin{aligned}
& u^{\varepsilon}(x, t)-u^{0}(x, t)-B(x, t, \varepsilon) \\
& =T(x, t)\left[v^{\varepsilon}(x, t)+v^{0}(x, t)-b^{\varepsilon}(x, t)\right]=T(x, t) z^{\varepsilon}
\end{aligned}
$$

by $(2.40)$. Then by Lemma $2,(2.41)$, we get

$$
\sup _{\substack{0 \leq x \leq 1 \\ 0 \leq t \leq T}}\left|u^{\varepsilon}(x, t)-u^{0}(x, t)-B(x, t, \varepsilon)\right| \leq c \varepsilon^{1 / 4} .
$$

The proof of the theorem is complete.

Acknowledgements. This work is contained in the doctoral dissertation written under the direction of Professor Peter D. Lax while the author was a graduate student at the Courant Institute of Mathematical Sciences, New York University. The author expresses his gratitude to him for several fruitful discussions. Also I thank the referee for the corrections which he suggested.

\section{REFERENCES}

1. C. Bardos, D. Brezis, and H. Brezis, Perturbations singulières et prolongments maximaux $D$ opérateurs positifs, Arch. Rational Mech. Anal. 53 (1973), 69-100.

2. C. Bardos and J. Rauch, Maximal positive B.V.P. as limits of singular perturbation problems, Trans. Amer. Math. Soc. 270 (1982), 377-408. 
3. R. Courant and D. Hilbert, Methods of mathematical physics, vol. 2, Wiley, 1975.

4. A. Friedman, Partial differential equations, Holt, Rinehart and Winston, 1969.

5. K. T. Joseph, Boundary layers in approximate solutions of initial boundary value problems for hyperbolic conservation laws, Ph. D. thesis, New York Univ., June 1987.

6. M. I. Visik and L. A. Lyusternik, Regular degeneracy and B.V.P. for linear differential equations with small parameter, Amer. Math. Soc. Transl. (2) 20 (1962), 239-364.

Tata Institute of Fundamental Research, 1.1. Sc. Campus, Bangalore-560012, India 